\title{
Dehiscencia del canal semicircular superior: Análisis de la producción científica
}

\section{Superior semicircular canal dehiscence: Analysis of scientific production}

\author{
Jesús Obón N¹, Ana Isabel Cisneros G1,2, Tania Gotor $\mathbf{S}^{1}$, Jaime Whyte $\mathbf{0}^{1,2}$.
}

\section{RESUMEN}

Introducción: La dehiscencia del canal semicircular es una vestibulopatía periférica rara y poco prevalente, que se caracteriza por una falta de cobertura ósea del canal semicircular superior en la zona más próxima a la duramadre de la fosa cerebral media.

objetivo: Conocer el estado actual y la calidad científica de las publicaciones sobre la dehiscencia del canal semicircular superior (DCSS).

Material y método: Se ha realizado una búsqueda bibliométrica con posterior revisión, selección y análisis a partir de ítems relacionados con estudios DCSS en la base de datos Pubmed desde el año 1998 hasta 2017.

Resultados: Al estudiar el tipo de publicaciones, el 77\% eran artículos originales, $12 \%$ revisiones, 9\% casos clínicos, 2\% cartas al director y respuestas al editor. Los artículos han sido publicados en un total de 108 revistas, siendo Otology and Neurotology la que mayor número de manuscritos presenta con un total de 87, seguida de Head and Neck Surgery con 28 y Laryngoscope con 22. Estados Unidos se posiciona como el principal contribuyente a la literatura mundial sobre este tema (42\%), seguido de Europa (33\%). El idioma de referencia es el inglés con 91\% de publicaciones (382). Según el índice de Lotka, la actividad productiva de los autores es de tipo medio/bajo, ya que de los 217 autores que firman en primer lugar, solo 19 tienen más de 10 artículos. Según el índice de impacto Journal Citation Reports, 60\% de las publicaciones se localiza en los cuartiles Q1 (116 publicaciones) y Q2 (141 publicaciones), lo que indica que la calidad de los trabajos es alta. La temática ha ido variando con el paso del tiempo, siendo actualmente el diagnóstico con $34,3 \%$, seguido del tratamiento con $25,7 \%$, los temas que más interesan. Aunque se trata principalmente de un tema del campo de la otorrinolaringología, en los últimos años ha despertado interés en otras áreas como la neurología y la radiología.

Conclusión: Este estudio revela como los trabajos sobre DCSS presentan un escaso número de autores, las publicaciones se concentran en pocas revistas, pero de una alta calidad, y el estado actual del tema está en fase de crecimiento exponencial.

Palabras clave: Dehiscencia del canal semicircular superior, análisis bibliométricos, factor de impacto, calidad científica.

\footnotetext{
1 Departamento de Anatomía e Histología Humanas, Universidad de Zaragoza, Zaragoza, España.

2 Grupo de DGA 40-17D, España.
}

Los autores declaran no tener conflictos de interés.

Recibido el 10 de enero, 2019. Aceptado el 5 de abril, 2019. 


\section{ABSTRACT}

Introduction: The dehiscence of the semicircular canal is a rare and not very prevalent peripheral vestibulopathy, characterized by a lack of bony coverage of the superior semicircular canal in the area closest to the dura of the middle cerebral fossa.

Aim: To know current status and scientific quality of publications of the superior semicircular canal dehiscence (SSCD).

Material and method: Bibliometric research with review, selection and analysis from items related with SSCD studies in the Pubmed database from 1998 to 2017.

Results: $77 \%$ of publications were original articles, $12 \%$ reviews, $9 \%$ clinical cases, $2 \%$ letters and answers to the editor. The articles have been published in 108 journals. The top publishing journal is Otology and Neurotology with 87 publications, followed by Head and Neck Surgery with 28 and Laryngoscope with 22. USA is the main global contributor to the world literature on this subject (42\%) followed by Europe (33\%). The publication reference language is English, with the 91\% of publications (382). According to Lotka's index, the general production activity of the authors is at the middle/low level. According to JCR impact factor, there are $60 \%$ of the publications in Q1 (116 articles) and Q2 (141 articles) quartiles, which indicates that the quality is high. The subject of the publications has varied over time, being currently diagnostic with $34.3 \%$, followed by treatment with $25.7 \%$, the subjects that most interest. In recent years it has aroused interest in other areas such as neurology or radiology.

Conclusion: The work on DCSS has a small number of authors, the publications are limited to a few journals, but of a high quality, and the current state of the subject is in phase of exponential growth.

Key words: Superior semicircular canal dehiscence, bibliometric analysis, impact factor, scientific quality.

\section{INTRODUCCIÓN}

En 1998, Minor y cols ${ }^{1}$ descubrieron una nueva forma de patología del oído interno que denominaron dehiscencia del canal semicircular superior. Se trata de una vestibulopatía periférica rara y poco prevalente a nivel internacional, que se caracteriza por una falta de cobertura ósea del canal semicircular superior en la zona más próxima a la duramadre de la fosa cerebral media. Este síndrome posee gran relevancia, desde el punto de vista histórico, ya que es, el único trastorno otológico descubierto y descrito en el área de otorrinolaringología a finales del siglo XX y que se ha desarrollado su estudio en el XXI. Ward y cols $(2017)^{2}$ realizaron con motivo de los 20 años de su descubrimiento una revisión de los conocimientos que actualmente se tienen sobre su origen, prevalencia, fisiopatología, variables clínicas, diagnóstico y procedimientos quirúrgicos curativos y futuras cuestiones, siendo la identificación de su etiología y la investigación de los posibles tratamientos médicos, para que no se produzcan alteraciones de la función del oído interno, las más significativas. Tras la búsqueda bibliográfica no encontramos ningún trabajo que analizara el impacto en la literatura mundial de las publicaciones sobre dicho síndrome.

\section{OBJETIVO}

Nuestro objetivo ha sido realizar mediante un estudio retrospectivo y descriptivo por medio de análisis bibliométrico. Dichos análisis tienen por propósito evaluar los manuscritos, su distribución y crecimiento, así como indagar sobre la repercusión de los autores y grupos de investigación que los producen.

\section{MATERIAL Y MÉTODO}

Se ha realizado un estudio descriptivo transversal de la producción científica sobre la dehiscencia del canal 
semicircular superior. Los datos se obtuvieron en la base de datos Medline ${ }^{3}$, (PubMed) desde marzo de 1998 hasta diciembre de 2017. Las palabras clave utilizadas en la búsqueda fueron: Superior semicircular canal dehiscence (437 manuscritos), semicircular canal dehiscence syndrome (230), semicircular canal dehiscence (514), superior canal dehiscence (476) y canal dehiscence syndrome (257). Una vez llevado a cabo la revisión bibliográfica (Pubmed) y leídos los trabajos, se han seleccionado 420 artículos. Las causas de exclusión del resto de artículos fueron: 1) no tratar el tema, aunque la temática fuera el canal semicircular superior, 2) citar solamente la dehiscencia como diagnóstico diferencial de otras patologías vestibulares y otológicas, 3) que versaran sobre dehiscencias de los otros canales semicirculares (posterior y lateral), y por último 4) aparecer publicados antes de 1998.

Con los manuscritos seleccionados confeccionamos una base de datos en Excel, en la que se recogen de cada uno de los artículos: autores, centro donde se realizó, el año de publicación del artículo, revista donde se publicó, el área a la que pertenece la revista, país de los autores, idioma de publicación del artículo, el impacto de la revista en el que se ha publicado según el Journal Citation Reports (JCR) y Scimago Journal Rank (SJR) ${ }^{5}$ que presenta la revista. Los factores de impacto son indicadores cuantitativos, miden la frecuencia con la que una revista ha sido citada en un año concreto y permiten comparar revistas, así como evaluar la importancia de ésta dentro de un campo científico. El valor del factor de impacto tiene la importancia de dar al trabajo una mayor difusión y incrementar su prestigio, y tienen como defecto que refleja más la calidad de la revista y no de los artículos publicados y varían con el tiempo tanto en número absoluto como en su posición, el tipo de trabajo y finalmente la temática de la que trata.

Para ver la calidad de las revistas que aparecen en JCR o SJR se ha utilizado su cuartil (Q1-Q4). Hemos considerado que una revista se considera clasificada como de alto impacto cuando está en el primer cuartil, medio impacto cuando está en el segundo y bajo impacto si se encuentra en el tercer o cuarto cuartil. Para seleccionar el cuartil de una revista hemos utilizado el factor de impacto correspondiente al año de su publicación.

En aquellas revistas que no tenían impacto JCR - SJR se buscó su ISSN (International Standard Serial Number) y se comprobó que no presentaban ningún factor de impacto. A partir de la base de datos, una vez ordenada, se realizó el análisis de todas las variables. Se analizó cada una de ellas de forma individual y, por otra parte, en los apartados que se podían, la comparación de dos variables diferentes.

Los indicadores a estudio han sido:

- Producción científica, número de artículos indexados en Pubmed.

- Tipología documental (original, revisión, caso clínico, cartas al director, etc.).

- Índice de productividad (logaritmo del número de trabajos originales o revisiones).

- Idioma de publicación del artículo.

- Distribución geográfica de procedencia de los artículos.

- Filiación institucional donde se ha realizado el artículo.

- Número de autores por artículo e índice de colaboración (cociente entre el número de firmas y el número de trabajos).

- Análisis del área de las revistas, donde se publicaron los artículos.

- Análisis de la temática del artículo (etiología, prevalencia, fisiopatología, clínica, diagnóstico, asociación con patología del oído u otras dehiscencias, y experimentales). La clasificación temática se ha realizado conjuntamente entre todos los autores del trabajo. Cuando ha surgido alguna discrepancia en el proceso de catalogación se sometía a discusión y se acordaba el apartado donde había que clasificarlo.

- Enlace y acceso (gratuito o de pago) al documento desde Pubmed.

- Identificación del artículo mediante código DOI (Digital Object Identifier).

- Impacto de la revista según Journal Citation Reports y Scimago Journal Rank.

- Ley de crecimiento de Solla-Price. Estudia el ritmo del crecimiento de la información científica distinguiendo 4 etapas o fases. Fase 1 de inicio del tema, fase 2 de crecimiento exponencial, fase 3 de crecimiento ralentizado y fase 4 de colapso del tema.

- Ley de productividad sesgada de Lotka. Analiza la producción de un científico según el número de sus publicaciones y los divide en tres niveles (pequeños productores con un solo trabajo, medianos productores entre 2 y 9 trabajos y grandes productores con 100 más trabajos). 
- Ley de dispersión o índice de Bradford. Esta ley nos permite averiguar la distribución y concentración de los diversos documentos en diferentes revistas que publican un mismo tema. Este índice distingue un núcleo específico que agrupa las revistas con un mayor número de artículos y varias zonas que contienen el mismo número de artículos que el núcleo y cuya concentración es menor.

\section{RESULTADOS}

\section{Producción científica}

De la consulta realizada en la base de datos Medline se obtuvieron un total de 420 referencias. La evolución de las publicaciones fue ascendente a lo largo de los años, ajustándose a un modelo lineal. Siendo el año 2017 el más productivo, con un total de 48 artículos $(11,42 \%)$ del total de publicaciones (Figura 1).

\section{Tipología documental}

El tipo de documento más frecuente fue el artículo original con 322 documentos $(76,6 \%)$, seguido por las revisiones $52(12,3 \%)$, casos clínicos 36 $(8,6 \%)$, cartas al director $8(1,9 \%)$ y respuestas al director $2(0,5 \%)$. El índice de productividad de los artículos originales fue de 2,52 y el de las revisiones 1,71 .

\section{Procedencia geográfica, idioma y filiación institucional}

Se han identificado documentos de 36 nacionalidades diferentes, siendo América del Norte donde mayor producción hemos localizado, con 190 referencias $(45,2 \%)$, seguido de Europa con 140 $(33,3 \%)$, Asia $51(12,1 \%)$, Oceanía $28(6,7 \%)$, Latinoamérica $6(1,4 \%)$ y África $5(1,2 \%)$. El país con mayor producción científica fue EE.UU. con 177 manuscritos, seguido por Alemania y Australia con 27. El idioma predominante fue el inglés con 382 artículos, seguido del alemán con 10 y el español y chino con 9. Se identificaron trabajos publicados en 156 instituciones, y éstas se clasificaron en tres niveles de rendimiento, extrapolando a las mismas el índice de Lotka. Ochenta y siete eran pequeños productores (1 ó 2 trabajos publicados), 60 medianos productores (entre 3 y 10 trabajos) y 8 grandes productores (más de 10 trabajos) que fueron Harvard Medical School (Massachusetts Eye and Ear), John Hopkins Hospital (Baltimore), David Geffen School of Medicine (Los Angeles), Hospital de Basurto (Bilbao), Departamento de Anatomía e Histología Humanas de la Universidad de Zaragoza, Karolinska Hospital (Estocolmo), Hospital Alma Mater Studiorum (Bolonia) y Royal Prince Alfred (Sydney).

\section{Autoría}

Un total de 1.848 autores se han contabilizado. A partir del análisis del número de trabajos por autor hemos observado que un total de 23 trabajos son publicados por un solo autor, 47 trabajos presentan dos autores, 82 por 3 autores, 101 por 4 autores, 55 por 5 autores, 45 por 6 autores, 35 por 7 autores, 16 por 8 autores, 6 por 9 autores, 7 por 10 autores, 1 por 11 autores, 1 por 13 autores y finalmente 1 por 15 autores. El índice de colaboración (1.848 firmantes/420 artículos) es de 4,4 y una mediana de 5 autores por trabajo. La evolución del número de autores fue ascendente a lo largo de los años. Cuando hemos calculado la

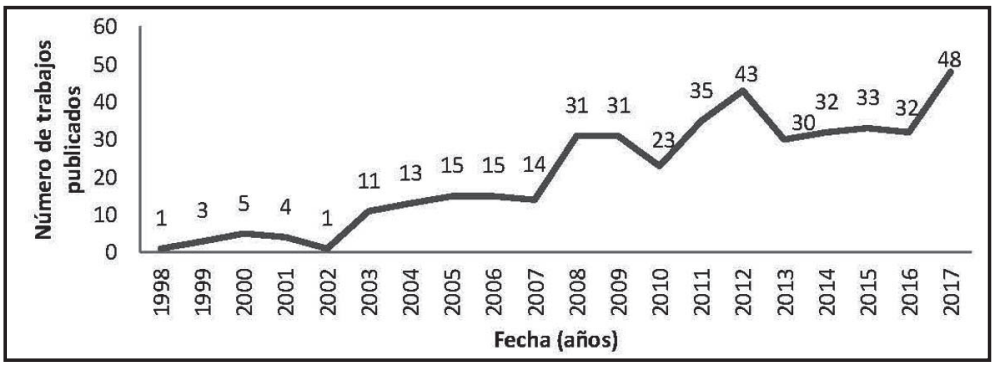

Figura 1. Distribución del número de publicaciones por año en DCSS. 
productividad de los autores por trabajo con índice de Lotka, esta es media/baja, solamente hemos observado 19 autores catalogados como grandes productores, 109 mediamos y 89 bajos.

\section{Revistas}

Los trabajos sobre dehiscencia del canal semi-circular superior están publicados en 108 revistas diferentes. Al analizar su concentración 3 revistas (137 artículos) componían el núcleo principal de Bradford (Otology and Neurotology (87), Otology Head Neck Surg (28) y Laryngoscope (22). La zona 1 estaba constituida por 14 revistas (135 artículos) y la zona 2 un de total de 91 revistas con 147 artículos. La distribución por áreas fue otorrinolaringología 47 , radiología 16 , neurología 13, medicina 12 neurocirugía 11 , odontología 2 , cirugía 1, pediatría 1, fisioterapia 1 , obstetricia y ginecología 1, oftalmología 1 y genética 1.

\section{Acceso al documento primario}

En relación al enlace del texto completo desde PubMed se vio como a 59 revistas se podían acceder de forma gratuita, 41 mediante diferentes medios de pago, y solamente en 8 no se permitía su consulta. Se comprobó como 340 publicaciones $(80,9 \%)$ estaban identificadas mediante DOI y 80 $(19,1 \%)$ no lo presentaban.

\section{Clasificación temática de la producción científica}

El tema sobre el cual se realizan mayor número de publicaciones ha sido el diagnóstico, con $34,3 \%$ de las publicaciones (144 trabajos). El segundo tema es el tratamiento, con $25,7 \%$ de las publicaciones (108 trabajos). También son temas relevantes a destacar etiología $(11,9 \%$ ) y clínica $(11,4 \%)$, con 50 trabajos y 48 trabajos respectivamente) (Figura 2).

\section{Indicios de calidad de las revistas}

De los 420 trabajos analizados $360(85,7 \%)$ presentan índice de impacto Journal Citations Reports (JCR) distribuidos de la siguiente manera: Q1 $116(27,61 \%)$, Q2 141 (33,57\%), Q3 53 (12,61\%), Q4 50 (11,90\%), y $60(14,3 \%)$ no tenían impacto. Cuatrocientos diez y siete trabajos aparecen en el índice Scimago Journal Rank (SJR): Q1 254 (60,47\%), Q2 80 (19,04\%), Q3 $58(13,80 \%)$ y Q4 $25(5,95 \%)$; solamente tres trabajos $(0,8 \%)$ no presentaron ningún indicio de calidad y estaban publicados en las revistas Cureus ISSN: 2168-8184, Laryngoscope Investig Otolaryngol ISSN: $2378-8038$ y Case Rep Otolaryngol ISSN: 2468-5488. Otros indicadores de calidad analizados fueron $\mathrm{H}$-index que es de 41, el número de trabajos que se consideran clásicos (más de 100 citas) que son 16, y el ESI Citation Report (Essential Science Indicators) con 250 trabajos (60\%) clasificados en el primer cuartil.

\section{Estado actual del tema}

El índice de Solla-Prince nos indica cómo los trabajos sobre dehiscencia de canal semicircular se encuentran en fase 20 de crecimiento exponencial, ya que en el periodo 1998 a 2007 se publicaron 82 manuscritos y en el de 2008 a 2017 un total de 338.

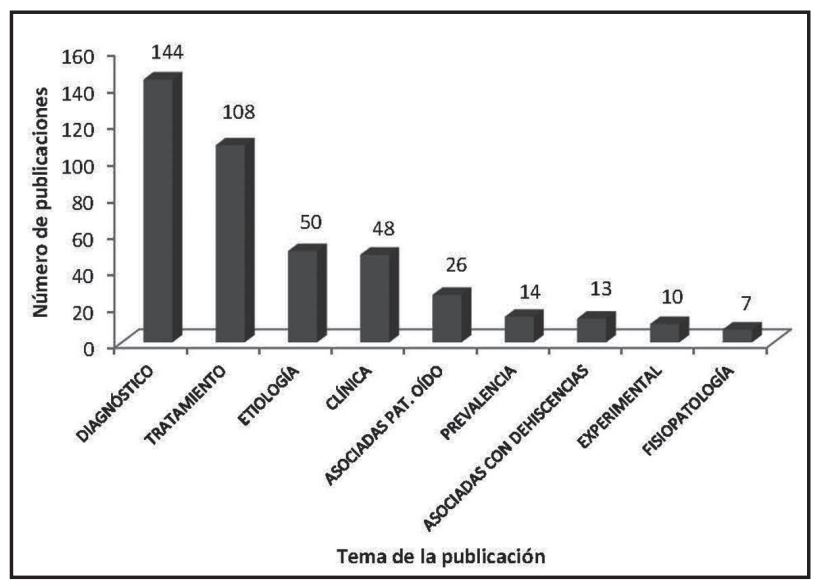

Figura 2. Clasificación temática y número de trabajos en DCSS. 


\section{DISCUSIÓN}

Los análisis bibliométricos tienen como objetivo fundamental estudiar y evaluar los documentos, su distribución y crecimiento, así como indagar sobre la repercusión de los autores y grupos de investigación que los producen. Esto nos ha llevado a realizar el análisis de la producción científica de las publicaciones sobre la dehiscencia del canal semicircular superior, aunque somos conscientes que al tratarse de una unidad pequeña su evaluación es difícil.

Hemos utilizado MEDLINE (PubMed) porque, por una parte, Bordons (2002) ${ }^{6}$ y Saunders (2017) la consideran la mejor y más completa base de datos biomédicos, y, por otra parte, por ser un buen instrumento para evaluar la calidad de las distintas fuentes, debido a la rigurosa selección de publicaciones que en ella aparecen según Martin-Rodero $(2016)^{8}$. Al hacer la búsqueda nos hemos encontrado con ciertas limitaciones siendo la más importante la inclusión de publicaciones no relacionadas directamente con el tema como hemos expuesto en el apartado de material y métodos. Al final, de los 514 posibles artículos se seleccionaron 420 artículos y se desecharon $94(18,2 \%$.) Este error en cuanto al número de publicaciones sobre el tema lo realiza Ward y cols $(2017)^{2}$ cuando en una revisión sobre los 20 años de la publicación de esta patología incluye una tabla tomada directamente de Pubmed, en la que aparecen más trabajos de los relacionados con el tema. Pensamos que una buena selección de las palabras claves y la no inclusión en los resúmenes del término dehiscencia del canal semicircular en los trabajos en los que no trata dicha patología, podrían evitar en parte este sesgo en la selección y mejorar su indexación.

Al realizar este manuscrito nos hemos hecho una serie de preguntas para determinar los aspectos más importantes de la evolución de la producción científica de este síndrome, sobre si la evolución es creciente 0 por el contrario, después de 20 años, empieza a ser un campo científico envejecido, si los trabajos sobre dehiscencia cumplen con las leyes de la bibliometría, en cuanto al crecimiento, concentración o dispersión y producción, y qué calidad tienen las revistas donde se publican trabajos sobre el tema.

La respuesta a la primera pregunta es que las publicaciones sobre dehiscencia han ido aumentando su crecimiento. Cuando se analiza este incremento agrupando los trabajos en periodos de 5 años, según sugiere Franchignoni y cols $(2011)^{9}$, hemos podido observar que la producción científica sobre DCSS sigue una línea creciente. En el periodo de 1998 al 2002, se publicaron 14 trabajos, aumentando a 68 entre 2003 y 2007, continúa el aumento a 163 manuscritos en el periodo de 2008 y 2012 y por último, entre 2013 y 2017 se noticiaron 175 artículos. Otro parámetro que refleja el interés por este tema, es el crecimiento lineal ascendente del número citas de estas publicaciones en Web of Science. Así por ejemplo en 2014 fueron 637 citas, en el 2015 de 732, en el 2016 aumentó a 787 y en el 2017 a 831 . Lo mismo ocurre con el índice h-índex que permite medir simultáneamente la calidad (en función del número de citas recibidas) y la cantidad de la producción científica. Este índice ha ido aumentando progresivamente desde 14 en el año 2000 hasta 41 al finalizar el año 2017. Estos datos nos indican que las publicaciones sobre DCSS no es un campo científico envejecido y sigue con un creciendo lineal y por lo tanto estando de actualidad.

Ante nuestra segunda cuestión y al estudiar las distintas leyes que rigen los análisis bibliométricos hemos observado que aplicando la ley obsolescencia de la literatura científica (Solla-Price) los trabajos sobre DCSS están en la actualidad en fase 2 (crecimiento exponencial), ya que cuando se analiza la producción científica en periodos de 10 años este tema no solo duplica el número de publicaciones como dice el enunciado de la ley, sino que las cuadriplica. En el periodo desde 1998 al 2007 se realizaron 82 trabajos mientras que de 2008 al 2017 fueron 338. Tendrán que transcurrir 10 años más para ver si las publicaciones siguen en crecimiento exponencial como hasta ahora, pueden pasar a la fase 3 de crecimiento ralentizado, 0 entran en fase 4 de colapso o saturación. El índice de Lotka, nos indica cómo la productividad de los autores que publican sobre la DCSS es de tipo media/baja, solo 19 grupos de investigación tienen más de 10 artículos. Estos datos son muy similares al comparar nuestros resultados con los que aparecen en Web of Science o Scopus.

Cuando hemos calculado el índice de productividad para los trabajos originales, éste fue de 2,52 mientras que para las revisiones fue del 1,71. Estas cifras son muy semejantes a las descritas por Sanz y Wanden (2017) ${ }^{10}$ de 2,31 para trabajos biomédicos. 
La Ley de dispersión o índice de Bradford nos indica que el núcleo principal está constituido por tres revistas (Otology and Neurotology, Otology Head Neck Surg y Laryngoscope), hecho que demuestra que los trabajos se concentran en muy pocas revistas. De estas tres revistas, Otology and Neurotology está focalizada preferentemente hacia el área de otoneurología mientras que la otras dos no presentan un enfoque predominante. El aumento de publicaciones sobre DCSS en Otology and Neurotology lo evalúan Boerner y cols (2017)11 donde describen el incremento de la publicación sobre este tema siendo sus hallazgos significativos $(p<0.001$ y $r=0,484)$.

De las tres leyes de la bibliometría estudiadas se concluye que los autores tienen una productividad media/baja, los artículos se concentran en muy pocas revistas y los trabajos en la actualidad presentan un crecimiento tipo exponencial. Como respuesta a nuestra tercera pregunta, sobre el prestigio y la calidad de la revista donde se publican los trabajos, se estudió mediante las bases de datos Web of Science (JCR) y Scimago (SJR) clasificando según, el cuartil donde figuraba el año de publicación. Hemos utilizado el cuartil y no el factor de impacto (FI) de la revista porque al ser una patología que ha sido estudiada por diferentes áreas (ORL, neurología, cirugía, radiología) en cada una de ellas los factores de impacto varían, habiendo áreas en el que el FI es muy alto como sucede por ejemplo en el área de neurología, y en otras más bajo como ORL. En cambio, el cuartil como indicador de la posición de una revista en relación con todas las de su área, nos permite darnos una idea más precisa de su relevancia como indica entre otros Trilla y cols $(2000)^{12}$.

Los resultados obtenidos analizando el índice JCR revelan cómo los trabajos sobre DCSS tienen una calidad alta, ya que el $60 \%$ de las publicaciones presentaron una posición en los cuartiles Q1 y Q2, y este porcentaje aumentó al 79,51\% con el índice SJR.

Desde el año 2013, Web of Science ha introducido un nuevo índice, ESI total citations, que evalúa en conjunto todas las revistas de área de biomedicina y que es útil cuando en un campo de la medicina hay pocas revistas, como sucede en el área de ORL 0 el tema a estudiar es multidisciplinar. En este índice, las revistas que más publican trabajos sobre DCSS están en el cuartil primero. Sirva de ejemplo Otology and Neurotology (revista que más publicaciones tiene sobre el tema de dehiscencia) que unos años aparece en el JCR como Q1 y otros como Q2, y en el ESI total citations siempre ha estado en el primer cuartil o European Archives of Oto-Rhino-Laryngology que con el JCR tiene un impacto Q2 y con ESI un Q1. Este índice avala la calidad alta de los trabajos sobre DCSS.

Estamos de acuerdo con Falagas y cols (2008) ${ }^{13}$ y Oosthuizen y Fenton $(2013)^{14}$ en que el Scimago Journal Rank (SJR) es un buen índice alternativo al JCR, al ser un recurso de acceso abierto, recoge la información de bases originales, enumera más títulos de revistas en una variedad más amplia de países e idiomas y la evaluación de la calidad de las citas, sobre todo cuando se utiliza el Source Normalised Impact per Paper (SNIP) y cuando se compara con el ESI el porcentaje de revistas en el primer cuartil este es muy similar. Como indica Beltran $(2006)^{15}$, el factor de impacto puede ser manipulado por la inclusión de documentos como notas editoriales, casos clínicos, o resúmenes de congresos, esto no sucede con los trabajos sobre dehiscencia de canal semicircular superior, ya que hemos observado cómo el tipo de publicación en un alto porcentaje eran originales $(76,6 \%)$, con un menor número de revisiones $(12,3 \%)$ y casos clínicos (8,57\%), las cartas al director $(1,9 \%)$ y respuestas de cartas al director son mínimas $(0,47 \%)$, y no hemos visto ningún resumen a congresos por lo que no influyen en el impacto.

Después de este análisis de los 3 aspectos que nos preocupaba dejar resueltos con este trabajo, queremos destacar que el aspecto original del mismo, y hasta ahora no publicado, ha sido catalogar las publicaciones según la temática del trabajo. Hemos observado que el tema sobre el cual se realizan mayor número de publicaciones ha sido el diagnóstico, con $35 \%$ (145 trabajos), seguido por el tratamiento, con $26 \%$ (108 trabajos). También son temas relevantes la etiología (51) y clínica (49), con $12 \%$. No hemos visto una correspondencia exacta entre las distintas áreas donde se publican los trabajos de DCSS en las bases Web of Science y Scopus. Mientras que en la primera muestra hay 25 áreas, en la segunda son 12. Algunas tan dispares a nuestro tema como arte y humanidades, agricultura y ciencias biológicas o ciencias sociales. Nosotros al ser la dehiscencia una patología multicéntrica donde intervienen distintas áreas y al igual que en los temas, hemos creado muestra propia catalogación con 11 apartados, destacando que el mayor porcentaje de trabajos se incluyen dentro del área de ORL con $38 \%$, seguido de las áreas mixtas 
que incluyen ORL-neurología y ORL-cirugía con 24\% y $11 \%$ respectivamente. Cabe subrayar como hay un número importante de manuscritos que se incluyen en el área de neurología (9\%) y radiología (5\%).

Como datos que nos han llamado la atención en este estudio son el aumento del número de publicaciones del tipo revisiones, como muestra de ello, en el 2017 de 48 trabajos publicados, 11 fueron revisiones, y que, con el paso de los años, este síndrome ha ido ganando relevancia en otras áreas que no son la otorrinolaringología, siendo la principal la neurología. Aunque por el diseño y metodología del trabajo no se ha estudiado las publicaciones sobre dehiscencia del

\section{BIBLIOGRAFÍA}

1. Minor LB, Solomon D, Zinreich JS, Zee DS. Sound and/or pressure induced vertigo due to bone dehiscence of the superior semicircular canal. Arch Otolaryngol Head Neck Surg 1998; 124: 249-58.

2. Ward BK, Carey JP and Minor LB. Superior Canal Dehiscence Syndrome: Lessons from the first 20 years. Front Neurol 2017; 8: 177 doi: 10.3389/ fneur.2017.00177.

3. MEDLINE: Pubmed [base de datos en Internet]. Available from: https://www.ncbi.nlm.nih.gov/ pubmed/

4. Web of Science [base de datos en Internet]. Disponible en https://www.fecyt.es/es/recurso/ web-science.

5. SCImago. SJR-SCImagojournal\& country Rank [base de datos en Internet]. Availablefrom: http:// www. Scimagogojr.com.

6. Bordons M, Fernández M, Gómez I. Advantages and limitations in the use of impact factor measures for the assesment of research performance in a peripheral country. Scientometncs 2002; 53, 195-206.

7. Saunders TFC, Rymer BC, McNamara KJ. A global bibliometric analysis of otolaryngology: Head and Neck Surgery literature. Clin Otolaryngol 2017; 42: 1338-42. canal semicircular superior en el año 2018, esta sigue una tendencia de crecimiento lineal con un total de 50 artículos y las revistas que más artículos siguen siendo Otology and Neurotology (6) y Laryngoscope (4).

\section{CONCLUSIÓN}

Este estudio revela como los trabajos sobre DCSS presentan un escaso número de autores, las publicaciones se concentran en pocas revistas, pero de una alta calidad, y el estado actual del tema está en fase de crecimiento exponencial.

8. Martín Rodero H. Revistas de Otorrinolaringología (ORL): Impacto y visibilidad. Rev ORL 2016; 7: 133-6.

9. Franchignoni F, Muñoz LaSA S. Bibliometric indicators and core journal in physical and rehabilitation medicine. J Rehabil Med 2011; 43: 471-6.

10. Sanz-Salero J, Wanden-Berghe C. Análisis bibliométrico de la producción científica, indizada en Medline, sobre los servicios de salud proporcionados por las unidades de hospitalización a domicilio. Hosp Domic 2017; 1: 21-34.

11. Boerner R, Hatch J, Harruff E, Nguyen S, Rizk H, Meyer T, LAMBERT P, McRackan T. Publishing trends in Otology and Neurotology. Otol Neurotol 2018; 39: 127-32.

12. Trilla A, Amymerich M, Carné X, Asenjo MA, Rodés J. Comparative analysis of articles published by Spanish authors (1993-1997) in biomedical journals with high impact factor. MedClin (Barc) 2000; 114: 609-13.

13. Falagas ME, Kouranos VD, Arencibia- Jorge R, Karageorgopoulos DE. Comparison of SCImago journal Rank indicator with journal impact factor. FASEB J 2008; 22: 2623-8.

14. Oosthuizen JC and Fenton JE. Alternatives to the important factor. Surgeon 2014; 12: 239-43.

15. Beltrán Galvis 0. Factor de impacto. Rev Col Gastroenterol 2006; 21: 57-61. 